\title{
Transcriptional induction of capsidiol synthesis genes by wounding can promote pathogen signal-induced capsidiol synthesis
}

Tomoya Kojima $^{1 \dagger}$, Nobuhide Asakura ${ }^{1 \dagger}$, Shiori Hasegawa ${ }^{1}$, Taishi Hirasawa ${ }^{1}$, Yuri Mizuno ${ }^{2}$, Daigo Takemoto ${ }^{2}$ and Shinpei Katou ${ }^{1 *}$ (D)

\begin{abstract}
Background: Plants are exposed to various forms of environmental stress. Penetration by pathogens is one of the most serious environmental insults. Wounding caused by tissue damage or herbivory also affects the growth and reproduction of plants. Moreover, wounding disrupts physical barriers present at the plant surface and increases the risk of pathogen invasion. Plants cope with environmental stress by inducing a variety of responses. These stress responses must be tightly controlled, because their unnecessary induction is detrimental to plant growth. In tobacco, WIPK and SIPK, two wound-responsive mitogen-activated protein kinases, have been shown to play important roles in regulating wound responses. However, their contribution to downstream wound responses such as gene expression is not well understood.

Results: To identify genes regulated by WIPK and SIPK, the transcriptome of wounded WIPK/SIPK-suppressed plants was analyzed. Among the genes down-regulated in WIPK/SIPK-suppressed plants, the largest group consisted of those involved in the production of antimicrobial phytoalexins. Almost all genes involved in the biosynthesis of capsidiol, a major phytoalexin in tobacco, were transcriptionally induced by wounding in WIPK/SIPK-dependent and -independent manners. 5-epi-aristolochene synthase (EAS) is the committing enzyme for capsidiol synthesis, and the promoter of EAS4, a member of the EAS family, was analyzed. Reporter gene analysis revealed that at least two regions each 40-50 bp length were involved in activation of the EAS4 promoter by wounding, as well as by artificial activation of WIPK and SIPK. Unlike transcripts of the capsidiol synthesis genes, accumulation of EAS protein and capsidiol itself were not induced by wounding; however, wounding significantly enhanced their subsequent induction by a pathogen-derived elicitor.

Conclusions: Our results suggest a so-called priming phenomenon since the induction of EAS by wounding is only visible at the transcript level. By inducing transcripts, not the proteins, of EAS and possibly other capsidiol synthesis genes at wound sites, plants can produce large quantities of capsidiol quickly if pathogens invade the wound site, whereas plants can minimize energy loss and avoid the cytotoxic effects of capsidiol where pathogens do not gain entry during wound healing.
\end{abstract}

Keywords: Disease resistance, MAPK, Phytoalexin, Priming, Wound

\footnotetext{
* Correspondence: shinpei@shinshu-u.ac.jp

${ }^{\dagger}$ Tomoya Kojima and Nobuhide Asakura contributed equally to this work.

${ }^{1}$ Faculty of Agriculture, Shinshu University, Nagano 399-4598, Japan

Full list of author information is available at the end of the article
}

(c) The Author(s). 2019 Open Access This article is distributed under the terms of the Creative Commons Attribution 4.0 International License (http://creativecommons.org/licenses/by/4.0/), which permits unrestricted use, distribution, and reproduction in any medium, provided you give appropriate credit to the original author(s) and the source, provide a link to the Creative Commons license, and indicate if changes were made. The Creative Commons Public Domain Dedication waiver (http://creativecommons.org/publicdomain/zero/1.0/) applies to the data made available in this article, unless otherwise stated. 


\section{Background}

In nature, various forms of environmental stress affect plant growth. Infection by pathogenic microbes is one of the most harmful stresses and can lead to the death of infected plants. Wounding caused by mechanical tissue damage or herbivory feeding also affects plant growth. Moreover, the effects of environmental stress are not independent but instead interact with each other. For example, wounding disrupts physical barriers present at the plant surface and increases the risk of pathogen invasion.

To protect themselves against pathogens, plants have developed a variety of defense mechanisms, which are separated into constitutive and inducible defenses (reviewed in [1]). Constitutive defenses include preaccumulated toxic chemicals and physical barriers such as epidermal cuticles and cell walls. Physical barriers restrict the invasion of most microbes, but they can be disrupted by pathogens, especially fungal pathogens, as well as by wounding. Inducible defenses are generally thought to be stronger than constitutive ones, but they are controlled to function only after the recognition of pathogens by plants, because induction of defense responses is associated with energy costs and some of them damage not only pathogens but also the plant itself. To detect pathogens, plants have acquired at least two systems that sense conserved or specific molecules of pathogens (reviewed in [2]). In the first system, conserved microbial molecules, called microbe-associated molecular patterns (MAMPs), are recognized by plant transmembrane pattern recognition receptors. In the second system, specific pathogen effectors, also known as avirulence proteins, are recognized by plant Resistance proteins. Once pathogens are detected, plants respond to them with inducible defenses such as the production of toxic chemicals, the expression of defense-related genes and often a rapid localized cell death, called the hypersensitive response. Plants can avoid energy loss and tissue damage by inducing strong defenses only after pathogen recognition.

Phytoalexins, low molecular weight antimicrobial compounds, are one of the best-known inducible defenses (reviewed in [3]). The structures of phytoalexins are very diverse, including terpenoids, phenylpropanoids, flavonoids, and alkaloid compounds, and they are not found in healthy tissues but are induced in response to pathogens and pathogen-derived elicitors. In tobacco (Nicotiana tabacum), the major phytoalexin is capsidiol, a bicyclic dihydroxylated sesquiterpene, whereas that in Arabidopsis (Arabidopsis thaliana) camalexin, an indole alkaloid compound, has the same role. Rice (Oryza sativa) produces a variety of diterpenoid phytoalexins as well as a flavonoid. The biosynthetic pathways of a number of phytoalexins have been clarified. For example, capsidiol is produced from isopentenyl diphosphate (IPP), a precursor of all isoprenoid compounds (Additional file 1: Figure S1). IPP is converted to farnesyl diphosphate (FPP), and then FPP is converted to capsidiol by the actions of 5-epi-aristolochene synthase (EAS) and 5-epi-aristolochene 1,3-dihydroxylase (EAH). 3-hydroxy3-methylglutaryl-CoA reductase (HMGR) catalyzes the rate-limiting step in the production of IPP (reviewed in [4]), whereas the functions of EAS and EAH are specific to capsidiol biosynthesis. Accumulation of phytoalexins is generally associated with the transcriptional activation of genes encoding their biosynthetic enzymes, and signaling pathways regulating the biosynthesis of phytoalexins are becoming clear.

Mitogen-activated protein kinase (MAPK) cascades, consisted of three interacting kinases, MAPK, MAPK kinase and MAPK kinase kinase, transduce various extracellular stimuli into intracellular responses (reviewed in $[5,6])$. Increasing evidence indicates that MAPK cascades control the production of phytoalexins. In Arabidopsis, a MAPK cascade consisting of MAPKKK $\alpha / M E K K 1, M K K 4 /$ MKK5, and MPK3/MPK6 regulates the pathogen-induced biosynthesis of camalexin $[7,8]$. In rice, a MAPK cascade consisting of OsMKK4 and OsMPK6 has been reported to regulate elicitor-induced accumulation of diterpenoid phytoalexins [9]. In tobacco, activation of WIPK and SIPK, pathogen- and wound-responsive MAPKs, induces the expression of a gene encoding HMGR [10]. It has also been shown that WIPK and SIPK are required for the expression of HMGR2 induced by pathogen infection in $N$. benthamiana [11].

MAPK cascades are activated and play important roles in wound responses too. We have shown that woundinduced generation of ethylene and jasmonic acid (JA), phytohormones regulating wound responses, is decreased by the suppression of WIPK and SIPK [12]. The $N$. attenuata MAPKs NaWIPK and NaSIPK were also reported to be required for wound-induced accumulation of JA [13]. These results indicate that WIPK and SIPK play important roles in the production of phytohormones mediating wound responses. However, their contribution to downstream wound responses such as gene expression is not well understood. In this study, we searched for genes whose expression is reduced in wounded leaves of WIPK/SIPK-suppressed plants. We show that almost all the genes involved in the biosynthesis of capsidiol were transcriptionally induced by wounding in WIPK/SIPK-dependent and -independent manners. Although wounding did not increase the levels of either capsidiol itself or EAS protein, the committing enzyme for capsidiol synthesis, it primed the later synthesis of capsidiol and EAS protein induced by a pathogen-derived signal, suggesting that the induction of capsidiol synthesis genes at the transcription level by 
wounding is a preventative reaction against possible invasion by pathogens at wound sites.

\section{Results}

Identification of genes down-regulated in WIPK/SIPKsuppressed plants by microarray analysis

To identify genes whose expression is regulated by WIPK and SIPK, transcripts that were down-regulated in wounded leaves of WIPK/SIPK-suppressed plants were searched for using a microarray. In tobacco, the levels of ethylene emission and JA peak 3-6 h and 6-12 h after wounding, respectively $[14,15]$. Therefore, total RNA was extracted from leaves at $9 \mathrm{~h}$ after wounding and subjected to microarray analysis. Of 43,759 oligo nucleotides probes set on the chip, 59 probes targeting 46 genes showed more than a 5 -fold decrease in WIPK/SIPK-suppressed plants compared with control plants (Additional file 2: Table S1). BLASTX searches of the NCBI database (http://blast.ncbi. nlm.nih.gov/Blast.cgi) were performed to predict putative functions of the target genes, and they were categorized into 14 classes according to a modified form of the classification described previously [16] (Fig. 1, Additional file 3: Table S2). Approximately half of the target genes were those involved in secondary metabolism. The second and third largest categories were "unknown" and "signal

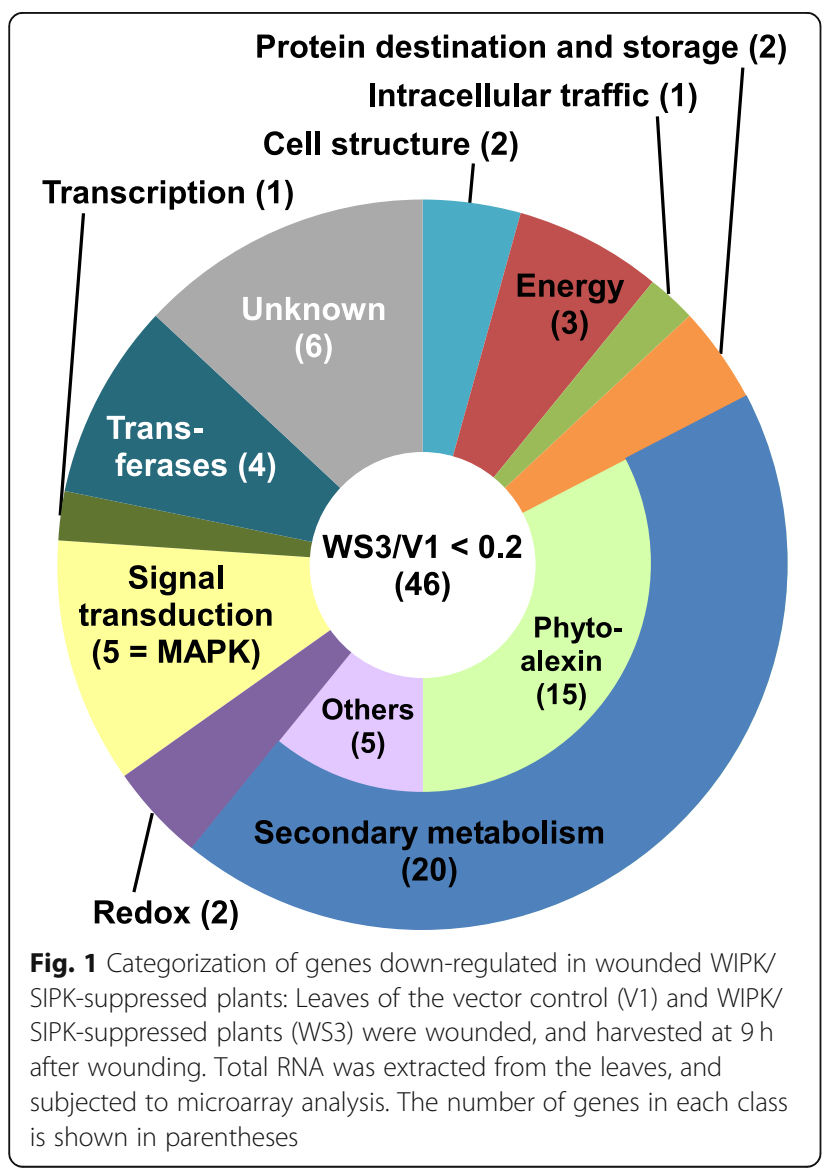

transduction", respectively. The five genes included in the "signal transduction" category were WIPK, SIPK and Ntf4, a close homolog of SIPK whose expression is suppressed in WIPK/SIPK-suppressed plants [12]. The remaining categories contained a few genes, and their predicted functions varied, indicating that WIPK and SIPK mainly regulate the expression of the genes involved in secondary metabolism.

Among the 20 genes categorized into secondary metabolism, 15 were predicted to be involved in phytoalexin synthesis (Fig. 1, Additional file 3: Table S2). Capsidiol is a major phytoalexin in tobacco and it is produced by the actions of EAS and EAH from FPP, an intermediate in the biosynthesis of many metabolites such as sterols, sesquiterpenes, triterpenes, and ubiquinones, as well as substrates for the farnesylation of proteins (reviewed in [4]) (Additional file 1: Figure S1). Many genes encoding EAS, $\mathrm{EAH}$, and their homologs were included in the list (Additional file 3: Table S2). To check the reproducibility of the microarray analysis, the transcript levels of $E A S$ and $E A H$ over a time course after wounding were analyzed by reverse transcription-quantitative PCR (RT-qPCR). Expression of $E A S$ and $E A H$ was strongly induced by wounding, with a peak around 9-12 $\mathrm{h}$ after wounding, and their transcript levels were decreased in WIPK/SIPK-suppressed plants (Fig. 2a). In contrast, the transcript levels of squalene synthase (SQS), another enzyme utilizing FPP as a substrate, were not significantly affected by the silencing of WIPK and SIPK, although it was also moderately induced by wounding.

\section{WIPK and SIPK regulate wound-induced expression of nearly all genes involved in capsidiol synthesis}

$E A S$ and $E A H$ were shown to be induced by wounding and regulated by WIPK and SIPK; therefore, we investigated whether other genes involved in capsidiol synthesis are regulated by WIPK and SIPK and whether they are induced by wounding. IPP, a precursor of FPP, is produced through the mevalonate pathway by the actions of six enzymes, and IPP is converted to FPP by IPP isomerase (IDI) and FPP synthase (FPS) (Additional file 1: Figure S1). Transcript analysis of 11 genes encoding any one of the enzymes revealed that all the genes except for FPS2 are clearly induced by wounding (Fig. 2b). In WIPK/SIPKsuppressed plants, transcript levels of all the genes except for HMGR1 and FPS2 were significantly decreased at least at one time-point in the experiments. Notably, no genes showed WIPK/SIPK dependency at $3 \mathrm{~h}$ after wounding, although approximately half of the genes were already induced by wounding at this time. Additionally, in case the enzymes are encoded by two paralogous genes (AACT, HMGR, and FPS), only one of two genes showed clear WIPK/SIPK-dependency. Similar results were obtained with another line of WIPK/SIPK-suppressed plants, ruling 

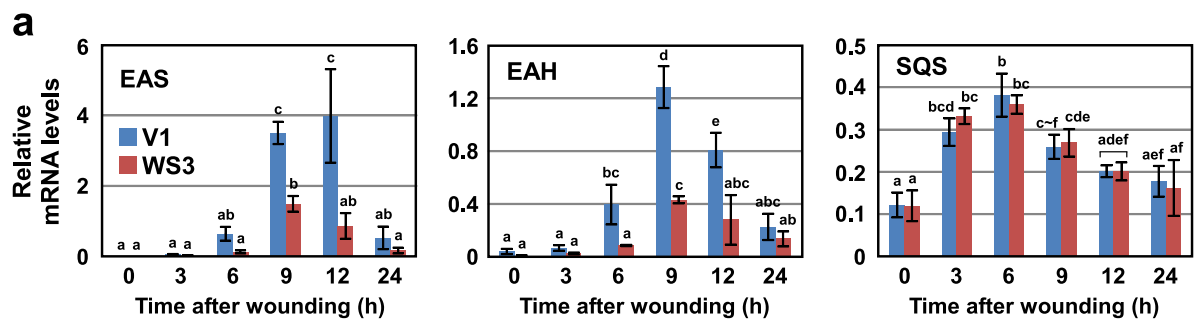

b
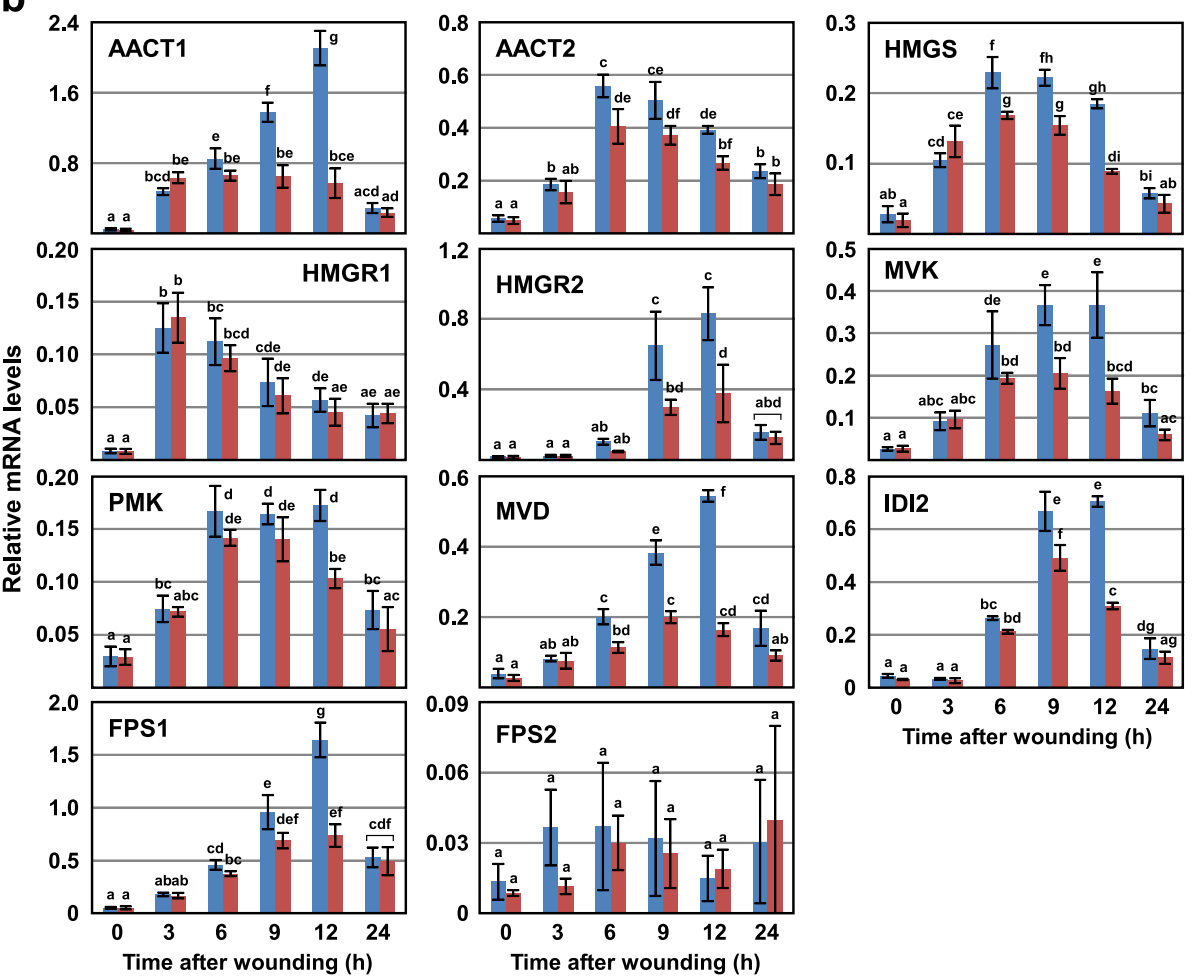

Time after wounding $(\mathrm{h})$

Fig. 2 WIPK and SIPK are involved in wound-induced expression of the capsidiol synthesis genes: $\mathbf{a}, \mathbf{b}$ Leaves of the vector control (V1) and WIPK/SIPK-suppressed plants (WS3) were wounded, and harvested at the times indicated after wounding. Transcript levels of EAS, EAH, and SQS (a), and genes of the mevalonate pathway (b) were quantified by RT-qPCR and normalized to the level of Actin2 as an internal standard. Values are means with standard deviations of three biological replicates. Significant differences among the groups were determined with one-way ANOVA followed by Tukey's HSD using KaleidaGraph 4.5 software. The lowercase letters at the top of the bars indicate significant differences $(P<0.05)$

out the possibility that this effect was caused by the introduction of the transformation vector (Additional file 4: Figure S2). These results indicated that induction by wounding of capsidiol synthesis genes is mediated by both WIPK/SIPK-dependent and -independent manners, and suggested that WIPK and SIPK regulate the expression of the specific members of gene families at relatively late time points.

IPP and dimethylallyl diphosphate, direct precursors of FPP, are produced not only in the mevalonate pathway but also in the so-called 2-C-methyl-D-erythritol 4-phosphate (MEP) pathway present in plastids (reviewed in [4]) (Additional file 1: Figure S1). Although it has been essentially considered that the two pathways function independently, some reports have indicated that interconnections exist between the pathways $[17,18]$. Therefore, we investigated the transcript levels of eight genes encoding any one of seven enzymes constituting the MEP pathway (Additional file 5: Figure S3). IDI1 was considered to be involved in the conversion between IPP and dimethylallyl diphosphate produced by the MEP pathway, because it encodes a protein with a putative plastid transit peptide (AB049815). Therefore, the transcript levels of IDI1 were also investigated. In contrast to the genes of the mevalonate pathway, all genes showed no or a very weak response to wounding, and none of the genes except for IDI1 showed WIPK/SIPK dependency. 
Both WIPK and SIPK are required for maximal induction of capsidiol synthesis genes

To investigate which of WIPK or SIPK is required for wound-induced expression of capsidiol synthesis genes, their transcript levels in WIPK- or SIPK-suppressed plants were quantified (Fig. 3). Although the transcript levels of the genes were generally decreased more by the silencing of SIPK than that of WIPK, single silencing of either WIPK or SIPK reduced the transcript levels of the most genes. These results suggested that WIPK and SIPK regulate the expression of capsidiol synthesis genes cooperatively, not redundantly.

\section{Promoter analysis of EAS4}

EAS is a committing enzyme for capsidiol production (Additional file 1: Figure S1). EAS4, a member of EAS gene family, is strongly induced by various forms of stress, and the responses of its promoter to pathogen-derived elicitor have been studied [19]. Therefore, EAS4 was chosen as a representative of capsidiol synthesis genes, and its promoter was analyzed to clarify how capsidiol synthesis genes are induced by wounding, and how WIPK and SIPK regulate them. Primers were designed based on database information, and an approximately 1.1-kbp EAS4 promoter region designated as 1126p was cloned (Fig. 4a). $1126 \mathrm{p}$ contains many sequence elements similar to the stress responsive cis-elements, but elements that mediate activation of the EAS4 promoter by elicitors have not been identified. The only functional element identified in the EAS4 promoter is a TAC-box. It was thought to function as a silencer or repressor, because the introduction of a mutation into the TAC-box increased the activity of the EAS4 promoter [20].

For the analysis of EAS4 promoter activity, we used an Agrobacterium-mediated transient expression in $N$. benthamiana leaves [21]. Agrobacterium cells carrying the EAS4 promoter fused to $\beta$-glucuronidase (GUS) as a reporter (EAS4p-GUS) were mixed with those carrying luciferase (LUC) driven by a Cauliflower mosaic virus $35 \mathrm{~S}$ promoter (35Sp-LUC) as an internal control of Agrobacterium infection, and then infiltrated into the leaves. Transcript levels of GUS, LUC, and Nbactin2 were quantified by RT-qPCR, and the level of GUS transcripts was doubly normalized to those of Nbactin2 and LUC. We first
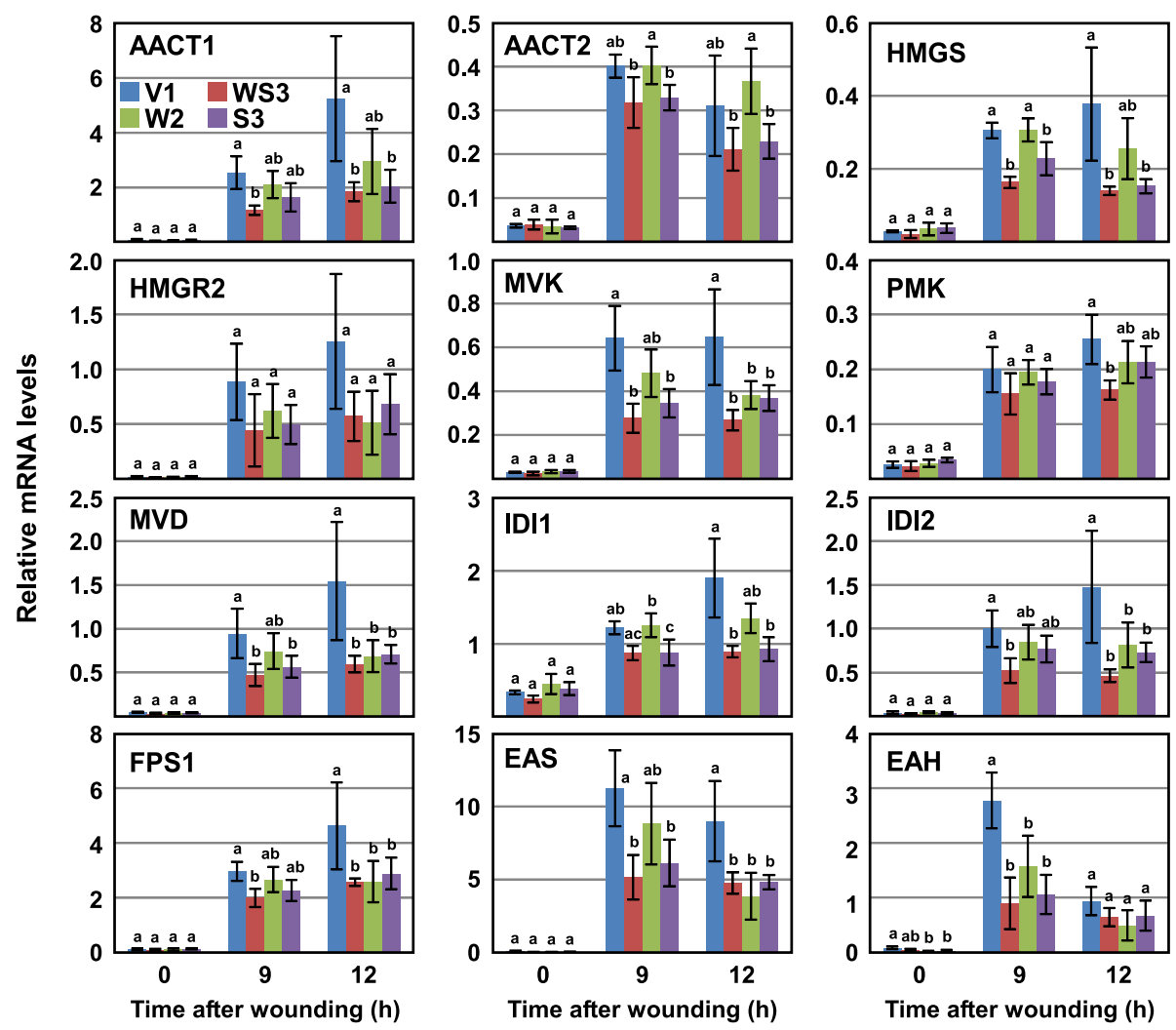

Fig. 3 WIPK and SIPK regulate the expression of capsidiol synthesis genes cooperatively: Leaves of the vector control (V1), WIPK/SIPK-suppressed (WS3), WIPK-suppressed (W2) and SIPK-suppressed (S3) plants were wounded, and harvested the times indicated after wounding. Transcript levels of the capsidiol synthesis genes were quantified by RT-qPCR and normalized to the level of Actin2 as an internal standard. Values are means with standard deviations of three to six biological replicates. Significant differences among the transgenic lines at each time point were determined with one-way ANOVA followed by Tukey's HSD using KaleidaGraph 4.5 software. The lowercase letters at the top of the bars indicate significant differences $(P<0.05)$ 


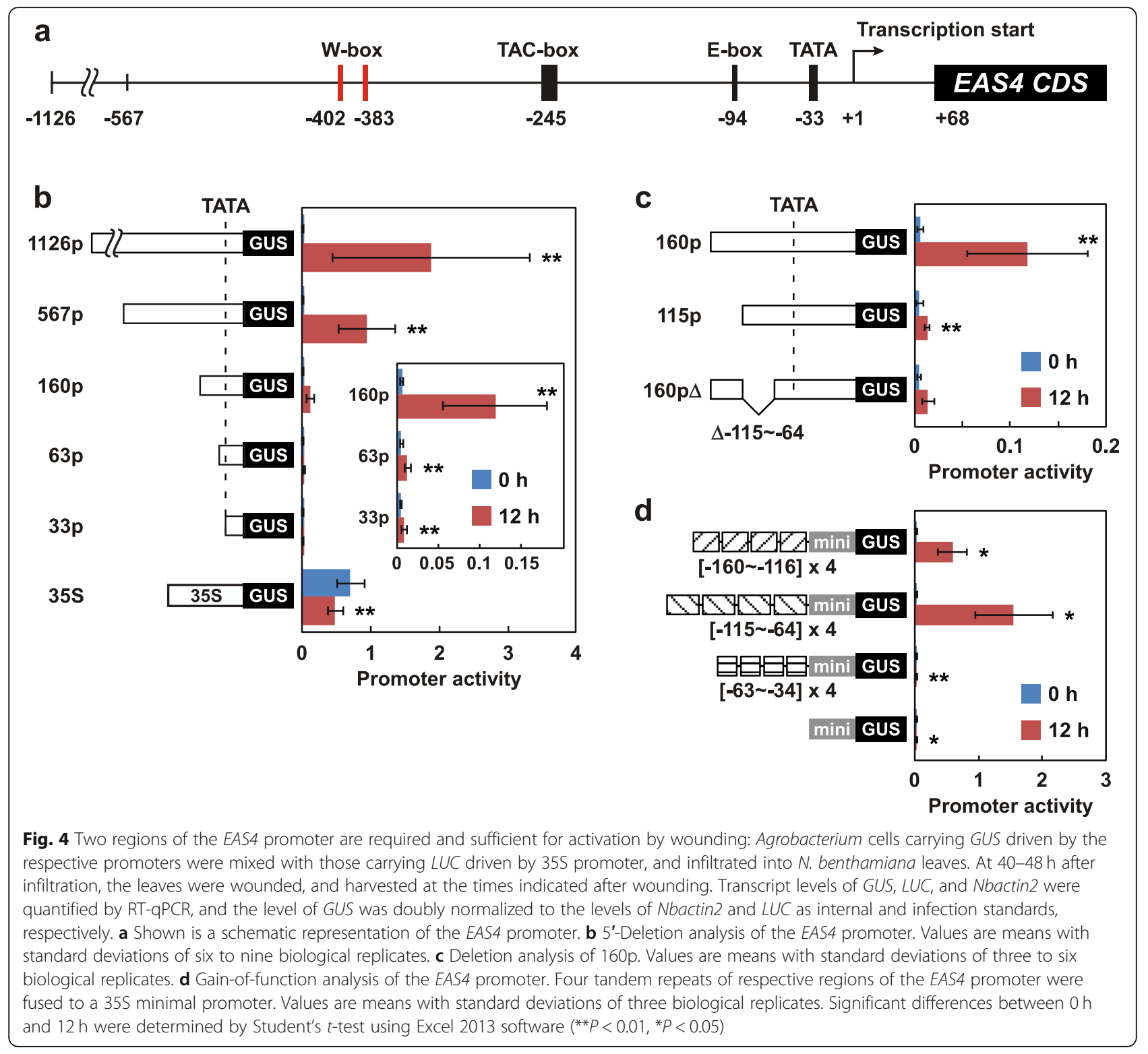

confirmed that 1126p is activated by wounding. As shown in Fig. 4b, the transcript level of GUS driven by $1126 \mathrm{p}$ was increased by wounding about 200-fold, reflecting about 170 -fold induction by wounding of the EAS transcript in tobacco (Fig. 2a). In contrast, the transcript levels of GUS driven by the $35 \mathrm{~S}$ promoter were not increased by wounding. Next, successive $5^{\prime}$-deletions of the EAS4 promoter designated as 567p (-567), 160p ($160), 63 p(-63)$, and $33 p(-33)$, were fused to GUS to identify the regions regulating wound responsiveness of the promoter. Deletion to -160 greatly decreased the activity of the promoter, but it was still activated by wounding more than 20-fold (Fig. 4b). Further deletion to -63 minimized wound-induced activation of the promoter, suggesting that a region from -160 to -64 is important for activation by wounding of the EAS4 promoter. The promoter fragments $63 \mathrm{p}$ and $33 \mathrm{p}$ still increased transcript levels of GUS slightly in response to wounding. However, it was considered to be an experimental artifact, because a $5^{\prime}$-untranslated region (UTR) of EAS4 and $35 \mathrm{~S}$ minimal promoter also showed results similar to 63p and 33p (Fig. 4d, Additional file 6: Figure S4).

To further delineate the region responsible for woundinduced activation, two deletion constructs of the 160p, $115 \mathrm{p}(-115)$ and $160 \mathrm{p} \Delta$, were created. An internal deletion construct $160 \mathrm{p} \Delta$ lacks a region from -115 to -64 . As shown in Fig. 4c, both constructs were hardly activated by wounding, suggesting that both regions from 160 to -116 and from -115 to -64 are required for 
wound-induced activation of 160p. The importance of regions from -160 to -116 and from -115 to -64 , but not a region from -63 to -34 , was further confirmed using a gain-of-function analysis. As shown in Fig. 4d, four tandem repeats of the regions from -160 to -116 and from -115 to -64 , but not the region from -63 to - 34, conferred strong wound-responsive activity on a $35 \mathrm{~S}$ minimal promoter.

\section{Mutational analysis of the promoter of EAS4}

To determine the regulatory elements in the region from - 160 to $-64,10$-bp substitutions were introduced into 160p (m1-m10, Fig. 5a). Substitution in any of the M2, M4, M5, M7, and M8 regions significantly decreased GUS transcript levels induced by wounding (Fig. 5b). In contrast, substitution in M1, M9, or M10 elevated GUS transcript levels induced by wounding. Without wounding, none of the substitutions affected GUS transcript levels. These results suggested that multiple woundresponsive cis-elements are present in a region from 150 to -81 of the EAS4 promoter.

\section{The EAS4 promoter is activated by MEK2 ${ }^{\mathrm{DD}}$, an activator of WIPK and SIPK}

Loss-of-function and gain-of-function analyses identified regions of the EAS4 promoter that are required and sufficient for activation by wounding (Figs. 4 and 5), but it was unclear if the activation is mediated by WIPK and SIPK or not. To induce activation of WIPK and SIPK specifically, we used $\mathrm{MEK} 2^{\mathrm{DD}}$, a constitutively active form of MEK2. MEK2 is an upstream MAPK kinase of WIPK and SIPK, and it directly phosphorylates and activates them [10]. As expected, the expression of MEK2 ${ }^{\text {DD }}$ activated the EAS4 promoter, although activation by MEK2 ${ }^{\mathrm{DD}}$ was weaker than that by wounding (Fig. 6a). These results supported that the EAS4 promoter is activated by both WIPK/SIPK-dependent and -independent mechanisms.

The EAS4 promoter contains two W-box-like sequences in a region from -410 to -310 (Fig. $4 a$ ). The W-box is a sequence recognized by WRKY transcription factors, and recent reports have indicated that WIPK and SIPK, and their orthologs in other plant species phosphorylate WRKY transcription factors and enhance their functions $[11,22,23]$. These lines of evidence prompted us to investigate the roles of W-box-like sequences in $\mathrm{MEK}^{\mathrm{DD}^{\mathrm{D}}}$-induced activation of the EAS4 promoter. Quantification of GUS transcript levels driven by a series of 5 '-deletions of the EAS4 promoter showed that the W-box-like sequences are dispensable for activation by MEK $2^{\mathrm{DD}}$ of the EAS4 promoter, and suggested that $160 \mathrm{p}$ is the shortest fragment required for activation by $\mathrm{MEK}^{\mathrm{DD}}$ (Fig. 6a). However, activation of $160 p$ by MEK $2^{\mathrm{DD}}$ was too weak to be concluded; therefore, gain-of-function analysis was

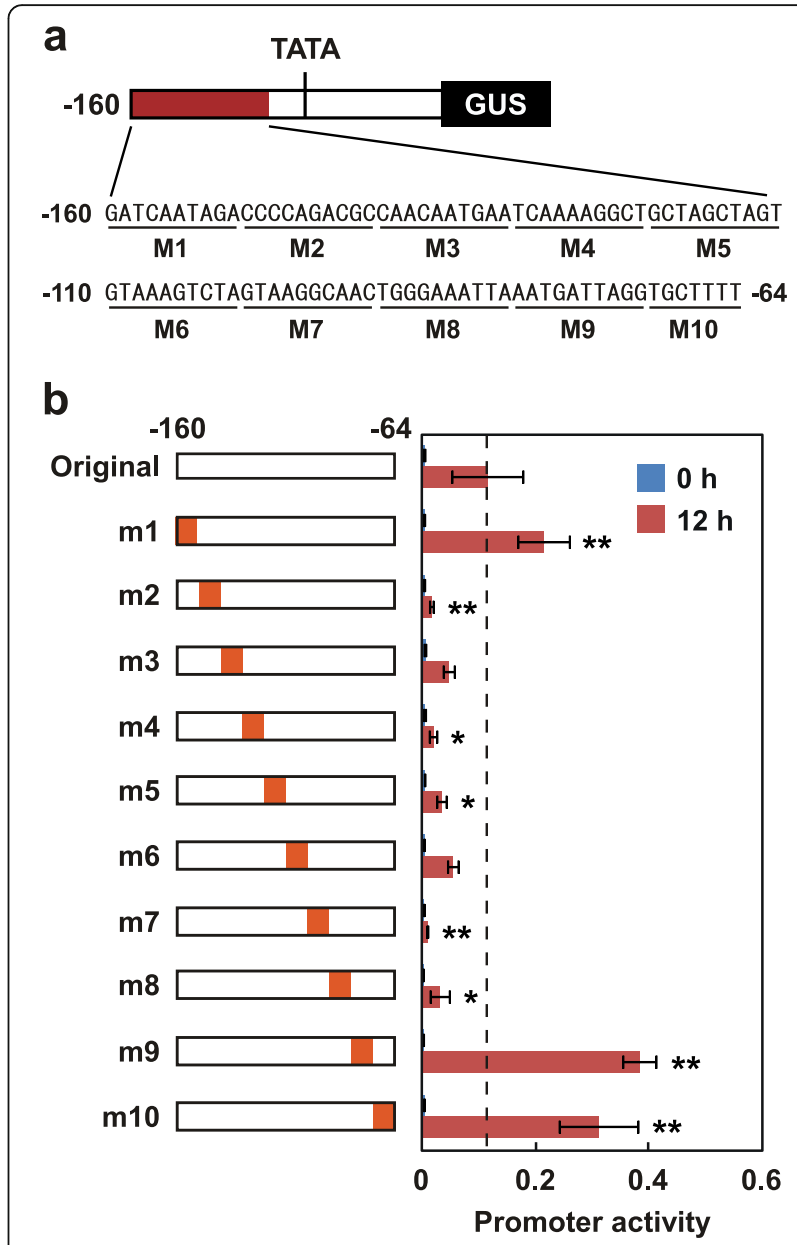

Fig. 5 Identification of nucleotide sequences of 160p required for its activation by wounding: a Shown is a schematic representation of $160 p$ and nucleotide sequences of the region from -160 to -64 of 160p. b Base substitution analyses of the region from -160 to -64 of 160p. Values are means with standard deviations of three-to-nine biological replicates. Significant differences between the original and m1 10 were determined by one-way ANOVA followed by Dunnett's test using KaleidaGraph 4.5 software $\left({ }^{*} P<0.01,{ }^{*} P<0.05\right)$

performed. As shown in Fig. 6b, tandem repeats of the regions from -160 to -116 and from -115 to -64 , but not the region from -63 to -34 , conferred MEK2 ${ }^{\mathrm{DD}}$-responsive activity on a $35 \mathrm{~S}$ minimal promoter. Moreover, tandem repeats of a region from -410 to -311 , which contains two W-box-like sequences, were activated by $M E K 2^{\mathrm{DD}}$. These results suggested that multiple regions of the EAS4 promoter are involved in its activation by WIPK and SIPK.

\section{Physiological roles of wound-induced expression of capsidiol synthesis genes}

It has been shown that most capsidiol synthesis genes are transcriptionally induced by wounding in WIPK/ SIPK-dependent and -independent mechanisms, and multiple regions of the EAS4 promoter are involved in 

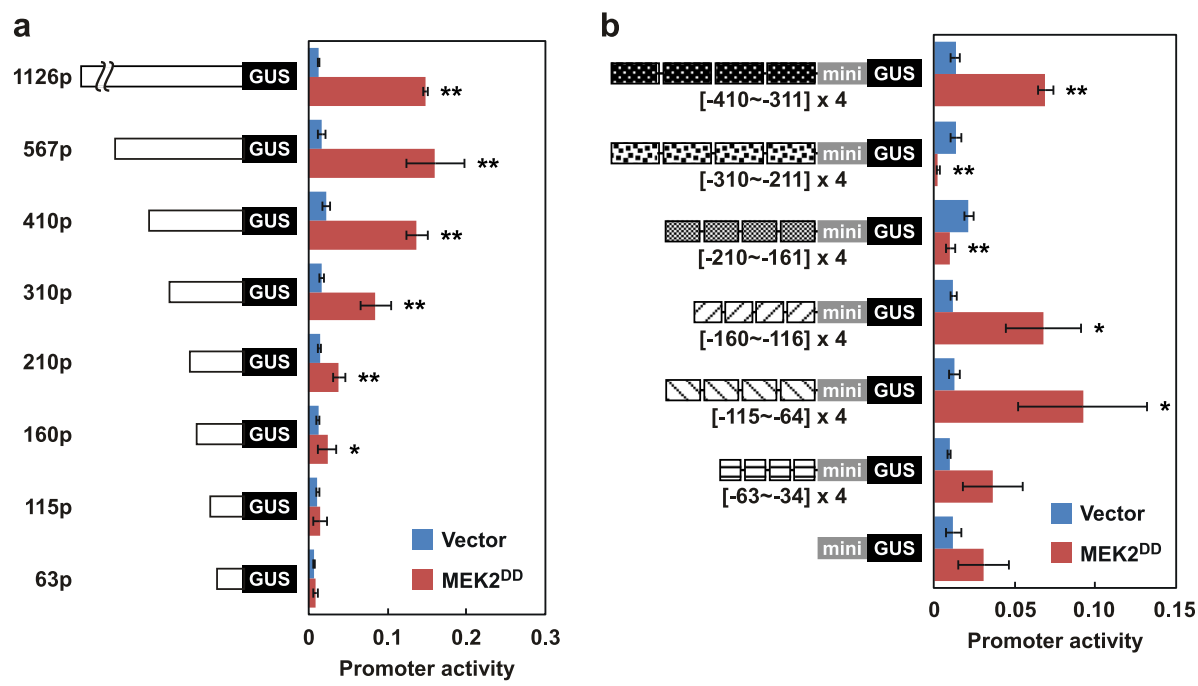

Fig. 6 Multiple regions of the EAS4 promoter are involved in activation by WIPK and SIPK: Agrobacterium cells containing GUS driven by EAS4 promoter fragments was mixed with those carrying LUC driven by a 355 promoter and those expressing MEK $2^{\mathrm{DD}}$ driven by a $35 \mathrm{~S}$ promoter, and then infiltrated into the leaves of $\mathrm{N}$. benthamiana. After incubation at $25^{\circ} \mathrm{C}$ for $48 \mathrm{~h}$, total RNA was extracted, and the transcript levels of GUS, LUC, and Nbactin2 were quantified by RT-qPCR. The levels of GUS were doubly normalized to the levels of Nbactin2 and LUC as internal and infection standards, respectively. a 5'-Deletion analysis of the EAS4 promoter. Values are means with standard deviations of three to six biological replicates. $\mathbf{b}$ Gain-of-function analysis of the EAS4 promoter. Four tandem repeats of the respective regions of the EAS4 promoter were fused to a $35 S$ minimal promoter. Values are means with standard deviations of three biological replicates. Significant differences between Vector and MEK2 $^{\mathrm{DD}}$ were determined by Student's $t$-test using Excel 2013 software $\left({ }^{* *} P<0.01,{ }^{*} P<0.05\right)$

its activation by wounding (Figs. 2, 4, and 6). These results indicated the importance of induction by wounding of capsidiol synthesis genes. However, as far as we know, no report has shown that accumulation of capsidiol is induced by wounding (similar to the majority of phytoalexins). We measured capsidiol levels in wounded tobacco leaves, but the levels were under the detection limit. Similarly, it has been reported that the accumulation of EAS protein is induced by a pathogen-derived elicitor, but scarcely by wounding in tobacco leaves [19]. We also confirmed that accumulation of EAS protein is induced by INF1, a protein elicitor secreted by Phytophthora infestans [24], but not by wounding (Fig. 7a).

Because wounding disrupts physical barriers in the leaf surfaces and causes a risk of pathogen invasion at the wound sites, it is reasonable to activate the biosynthesis of capsidiol at the wound sites during wound healing. However, it costs energy to produce capsidiol, and phytoalexins including capsidiol are toxic not only to pathogens but also to the plant themselves $[25,26]$. Therefore, in case pathogens do not enter the plant during wound healing, the production of capsidiol results in loss of energy and unnecessary damage to plant tissues. These lines of evidence suggest that induction by wounding of transcript levels, not protein levels, of EAS is a preventive response against possible invasion by pathogens at wound sites. If pathogens enter the wound, plants can synthesize EAS protein quickly, which leads to a rapid production of capsidiol, whereas if pathogens are not present, plants can minimize energy loss and avoid damage to the cells by capsidiol. To test this hypothesis, we investigated whether pre-wounding increases the levels of EAS protein and capsidiol induced by INF1.

In preliminary experiments, we found that it is technically difficult to infiltrate INF1 solution into wounded sites of leaf discs. Therefore, two different methods were tested to wound the leaves. In the first method, small holes were made in the leaves by pricking with a $10-\mu \mathrm{l}$ tip (hole method). In the other method, the leaves were crushed by holding with forceps strongly (crush method). Both methods clearly induced the expression of EAS (Additional file 7: Figure S5), and INF1 solution infiltrated relatively easily into the tissue damaged by the crush method, but not by the hole method. Therefore, leaves were wounded by the crush method, and INF1 was infiltrated into the damaged area at $9 \mathrm{~h}$ after wounding, at which time the accumulation of EAS transcript peaks (Figs. 2a, 3). As shown in Fig. 7b, the levels of EAS protein induced by INF1 were, as expected, increased by pre-wounding. Similarly, INF1-induced capsidiol production was enhanced by pre-wounding (Fig. 7c). At $6 \mathrm{~h}$ and $7.5 \mathrm{~h}$ after INF1 treatment, the levels of capsidiol were approximately doubled by pre-wounding. The effect of pre-wounding became less clear at $9 \mathrm{~h}$ after wounding, probably due to transcriptional activation by INF1 of EAS and other capsidiol synthesis genes. 

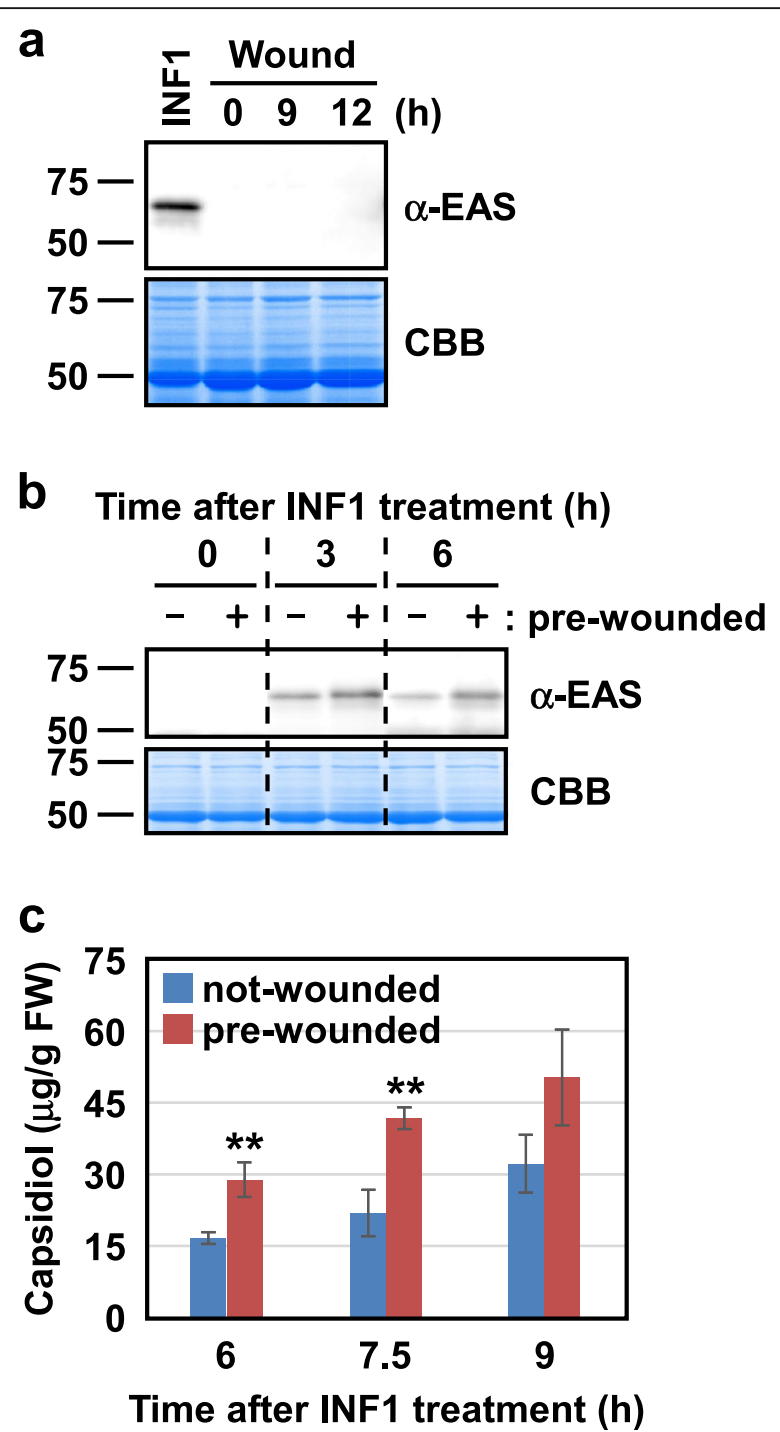

Fig. 7 Wounding enhances subsequent induction of EAS protein and capsidiol by INF1: a Leaves of the wild type tobacco were wounded or infiltrated with $25 \mathrm{nM}$ INF1, and harvested the times indicated. The accumulation of EAS protein was investigated by immunoblotting analysis using an anti-EAS antibody (a-EAS). As a loading control, parallel gels were stained with Coomassie Brilliant Blue R-250 (CBB). b, c Leaves were infiltrated with 25 nM INF1 directly or at $9 \mathrm{~h}$ after being wounded by holding the leaves with forceps. The samples were harvested at the times indicated after INF1 treatment, and the accumulation of EAS protein (b) and capsidiol (c) was investigated by immunoblotting analysis using an anti-EAS antibody (a-EAS) and HPLC, respectively. b As a loading control, parallel gels were stained with Coomassie Brilliant Blue R250 (CBB). c Values are means with standard errors of five or six biological replicates. Significant differences between not-wounded and pre-wounded were determined by Student's t-test using Excel 2013 software $(* * P<0.01)$

\section{Discussion}

Here, we showed that the expression levels of almost all genes involved in capsidiol synthesis are induced by wounding in WIPK/SIPK-dependent and -independent manners (Figs. 2, 4). Although WIPK and SIPK share high sequence homology and an upstream MAPK kinase, they likely function cooperatively, but not redundantly, because the induction of capsidiol synthesis genes was reduced by the suppression of either WIPK or SIPK (Fig. 3). Similar results were reported in the regulation of ethylene and camalexin synthesis by MPK3 and MPK6, Arabidopsis orthologs of WIPK and SIPK [7, 27]. Induction of capsidiol synthesis genes is reduced, but not lost, in WIPK/SIPK-suppressed plants; especially at early time points, and the effect of WIPK/SIPK-suppression was negligible (Fig. 2). Moreover, activation of the $E A S 4$ promoter by MEK $2^{\mathrm{DD}}$ was much weaker than that by wounding, although $\mathrm{MEK} 2^{\mathrm{DD}}$ and wounding targeted similar regions of the EAS4 promoter (Figs. 4, 6). These results suggested that the MAPK-pathway and other signaling pathway(s) cooperatively mediate the induction by wounding of capsidiol synthesis genes. One candidate for such signaling pathways is a pathway consisted of $\mathrm{Ca}^{2+}$ and $\mathrm{Ca}^{2+}$-regulated kinases. In rice cultured cells, suppression by RNA interference of OsCIPK14 and OsCIPK15, two $\mathrm{Ca}^{2+}$-regulated kinases, partially reduced the accumulation of phytoalexins and the expression of their biosynthesis genes induced by pathogen-derived elicitors [28]. Involvement of $\mathrm{Ca}^{2+}$ and $\mathrm{Ca}^{2+}$-regulated kinases in the activation of the EAS4 promoter should be a subject of future analyses. In WIPK/SIPK-suppressed plants, the emission of ethylene and accumulation of JA induced by wounding were reduced [12]. Because the EAS4 promoter is not activated by methyl jasmonate, a methyl ester form of JA [19], and expression of $E A S$ and $E A H$ is induced by ethylene treatment in $N$. benthamiana $[29,30]$, WIPK and SIPK likely induce the expression of $E A S, E A H$ and possibly other capsidiol synthesis genes via ethylene biosynthesis. Notably, MPK3 and MPK6 regulate the biosynthesis of both ethylene and camalexin, an indole-type phytoalexin, but the production of camalexin and expression of its biosynthesis gene are independent of ethylene [7]. These results suggested that MAPKs regulate the production of different types of phytoalexins by distinct mechanisms. In contrast to capsidiol synthesis genes, wound-induced expression of $S Q S$ was not significantly affected by the silencing of WIPK and SIPK (Fig. 2a). A recent report showed that a WRKY transcription factor, WsWRKY1, directly bound to the promoter of $S Q S$ and activated its transcription in Withania somnifera [31]. Interestingly, WsWRKY1 activated the expression of not only $S Q S$ but also mevalonate pathway genes such as HMGR. The tobacco homolog of WsWRKY1 might be involved in wound-induced expression of $S Q S$ and capsidiol synthesis genes in WIPK/SIPK-suppressed plants.

Reporter gene analysis revealed that 160p is a minimum promoter to respond to wounding (Fig. 4b). Deletion and 
mutation analyses of $160 \mathrm{p}$ indicated that the whole region spanning from -150 to -81 is essential for the promoter activity (Figs. 4c, 5). In contrast, gain-of-function analysis using four tandem repeat constructs with the indicated regions -160 to -116 and -115 to -64 independently conferred strong wound responsivity on a $35 \mathrm{~S}$ minimal promoter (Fig. 4d). Analysis of the MEK2 ${ }^{\mathrm{DD}}$ responsiveness of the EAS4 promoter showed similar results (Fig. 6). We have no clear explanation for this discrepancy, but one possibility is that a transcription factor regulating wound-induced expression of EAS4 might require at least two binding sites to form a stable complex on the promoter. Supporting this hypothesis, the nucleotide sequences of regions -149 to -140 and -96 to -87 are similar to each other in opposite directions (Additional file 8: Figure S6). We tried to detect such a transcription factor by electrophoretic mobility shift assays using several fragments of 160p labeled with biotin and nuclear proteins extracted from wounded leaves, but no band shift corresponding to activation by wounding was observed. Newman et al. [20] also failed to detect transcription factors that mediate the activation of the EAS4 promoter using a pathogen-derived elicitor. In the region -150 to -81 , no wound responsive cis-element is predicted, but an E-box (CANNTG) motif is present in the region -94 to -89 . The $E$-box sequence is recognized by bHLH-type transcription factors and is involved in responses to environmental stress such as salt stress [32]. In addition, ethylene signaling is thought to play important roles in INF1-induced expression of EAS [29], and many transcription factors such as ethylene response factor (ERF) function downstream of ethylene signaling. Very recently, it was shown that ERF2-like, an ERF-like protein, binds directly to and activates the promoter of NaEAS12, a member of EAS family in $N$. attenuate [33]. Our microarray analysis also showed that the transcript levels of ERF-like are decreased in WIPK/SIPK-suppressed plants (Additional file 3: Table S2). Investigation of these transcription factors should be a subject of future analyses.

In comparison to direct treatment of INF1, production of EAS protein and capsidiol was increased when leaves were treated with INF1 after wounding (Fig. 7). This result suggested that the induction of capsidiol synthesis genes by wounding only at the transcript levels is probably a priming phenomenon. Priming enables not only faster and stronger production of capsidiol against pathogens invading the wound site but also minimizes energy loss and damage by capsidiol in the absence of pathogen attack during wound healing. Of note, Chassot et al. [34] reported that priming of camalexin production by wounding in Arabidopsis is induced by a distinct mechanism. They showed that wounding hardly induces the expression of camalexin synthesis genes, but instead it primes their expression and camalexin production induced by Botrytis cinerea. These results suggested that distinct mechanisms underlie the priming of different types of phytoalexin. Moreover, we found that flg22, an epitope of bacterial MAMP flagellin [35], also induced accumulation of the EAS transcript, but it hardly affected EAS protein or capsidiol levels. MAMPs including flg22 are common to many microbes; therefore, if plants produced capsidiol in response to the MAMPs, capsidiol production will be induced not only by pathogens but also by non-pathogenic and beneficial microbes, which may result in disadvantageous and detrimental effects on plants. Similar to the case of wounding, plants might beware of unidentified microbes by inducing the transcripts, not the proteins, of capsidiol synthesis genes, which minimizes energy loss and avoids the cytotoxic effects of capsidiol when the microbes are not pathogens. Currently, it is unclear whether other capsidiol synthesis genes are regulated in a similar manner to EAS. Regulation of phytoalexin synthesis enzymes has been studied almost exclusively at the transcriptional level; however, some reports have suggested that they are also regulated at the post-transcriptional level. In potato tubers treated with a pathogen-derived elicitor, the transcript levels of $h m g 2$ and $h m g 3$ encoding HMGR remain high even after HMGR activity returned to the basal level [36]. In potato and oat, the expression levels of phytoalexin synthesis genes are induced similarly by both incompatible and compatible races of pathogen; however, high levels of phytoalexin are specifically induced by incompatible races [37, 38]. Moreover, Keller et al. [39] reported that EAS activity is not necessarily rate-limiting for capsidiol production. These lines of evidence suggested that posttranscriptional regulation of capsidiol synthesis genes other than $E A S$ might play important roles in the regulation of capsidiol production.

The mechanisms controlling the translation of EAS transcripts are currently unknown. Previous studies have revealed that most elements controlling the translation of specific transcripts are located within the $5^{\prime}$ - and $33^{\prime}$ UTRs of transcripts [40]. Xu et al. [41] reported that an R-motif, consisting of mostly purines, in the $5^{\prime}$-UTR increases translational efficiency of pattern-triggered immunity-associated genes. In contrast, it was reported that ethylene-induced translational regulation of $E B F 2$, a negative regulator of ethylene signaling, is mediated by its 3'-UTR [42]. The mechanisms that regulate the translation of broader, non-specific transcripts also exist. Ohtsu et al. [43] reported that silencing of NbNup75, encoding a nuclear pore protein nucleoporin 75 , increases the nuclear accumulation of polyA RNA. It will be of interest to test whether the UTRs of EAS and nucleoporin-mediated mRNA transport are involved in the translational regulation of EAS transcripts. By elucidating the mechanisms underlying the translational 
regulation of EAS transcripts, we will understand how plants produce capsidiol against pathogens quickly while minimizing energy loss and avoiding damage caused by the production of capsidiol.

\section{Conclusions}

In this study, we suggested that the induction of capsidiol synthesis genes by wounding only at the transcriptional level is a so-called priming phenomenon. By inducing the transcripts, not the proteins, of capsidiol synthesis genes at wound sites, plants can produce large quantities of capsidiol quickly if pathogens invade the wound site, whereas plants can minimize energy loss and avoid the cytotoxic effects of capsidiol where pathogens do not gain entry during wound healing.

Plant responses against pathogens and wounding have been investigated separately in most studies. However, the effects of pathogen infection and wounding are not independent but instead interact with each other; wounding disrupts physical barriers present at the plant surface and increases the risk of pathogen invasion. Therefore, plants have evolved sophisticated mechanisms to cope with the interacting effects of wounding and pathogen infection. The findings reported herein contribute to our understanding of such plant defense mechanisms.

\section{Methods}

\section{Plant materials and plant growth conditions}

The tobacco (Nicotiana tabacum) cultivar Samsun NN containing the $N$ gene and $N$. benthamiana were used. Their seeds were originally obtained from Leaf Tobacco Research Center, Japan Tobacco. The generation of SIPK-, WIPK-, and WIPK-and-SIPK-silenced tobacco plants has been described previously [12]. Plants were grown in pots containing vermiculite in a chamber maintained at $25^{\circ} \mathrm{C}$ with $16 \mathrm{~h}$ of light. The fully expanded leaves of 5-6-week-old plants of tobacco and $N$. benthamiana were used for experiments.

\section{Wounding treatment}

Unless otherwise stated, wounding treatment was performed by excising discs from leaves using a cork borer (diameter $10 \mathrm{~mm}$ ). The leaf discs were floated on water and incubated at $25^{\circ} \mathrm{C}$. In Fig. 7, leaves were wounded by two other methods. In the first method, by pricking with $10-\mu \mathrm{l}$ tip, one or four small holes per a $1-\mathrm{cm}$ diameter circle were made in the leaves connected with a plant body. In the other method, the leaves connected with a plant body were crushed by holding with forceps strongly.

\section{Preparation and treatment of INF1}

Recombinant protein of INF1 was prepared as described previously [24, 29]. INF1 solution $(25 \mathrm{nM})$ was infiltrated into the intercellular spaces of the leaves using a needleless syringe. To open stomata, plants were exposed to high humidity in the light for about $30 \mathrm{~min}$ prior to infiltration of INF1.

RNA extraction, microarray analysis, and RT-qPCR analysis Total RNA was extracted using TRIzol reagent (Thermo Fisher Scientific, USA) in accordance with the manufacturer's instructions. Microarray analysis was performed as described previously [44]. The analysis was performed once, and the data have been deposited in the GEO repository with the accession code GSE133681. The putative functions of the transcripts down-regulated in WIPK/SIPK-suppressed plants were predicted as described previously [44], and categorized into 14 classes according to a modified form of the classification described previously [16].

RT-qPCR analysis was performed using a SYBR PrimeScript RT-PCR Kit II (Takara, Japan). The relative expression level of each gene of interest was calculated as $2^{- \text {(CTgene of interest-CTreference })}$. Actin2 and Nbactin2 were used as reference genes. They were chosen from among three candidate genes, of which two encode actin and one encodes glyceraldehyde 3-phosphate dehydrogenase [44]. Primer pairs are listed in Additional file 9: Table S3.

\section{Cloning of the EAS4 promoter and plasmid construction}

Primers were designed based on information in the database [19]. The EAS4 promoter fragment was amplified by PCR with the primers using genomic DNA from healthy tobacco leaves as a template. EAS4 promoter fragments containing -1126 to +67 (just before the start codon) were amplified by PCR with HindIII and BamHI sites attached to the $5^{\prime}$ - and $3^{\prime}$-ends, respectively, and cloned into the corresponding sites of pBluescript II SK (+) (X52328). 5'-Deletion constructs were constructed by PCR using primers with HindIII site at their $5^{\prime}$-end. Internal deletions and base substitutions were introduced by inverse PCR using KOD -Plus- Mutagenesis Kit (Toyobo, Japan). Primer pairs used for deletion and substitution of the EAS4 promoter are listed in Additional file 9: Table S3.

To prepare tandem repeat constructs, subsets of EAS4 promoter fragments were amplified by PCR with HindIIISalI and XhoI sites attached to the $5^{\prime}$ - and $3{ }^{\prime}$-ends, respectively, and cloned into HindIII and XhoI sites of pBluescript II SK (+), generating pBS2-HindIII-SalI-EAS4 promoter fragment-XhoI. The promoter fragment was obtained as a HindIII-XhoI fragment from the construct and cloned into the HindIII-SalI sites of the same construct, resulting in two tandem repeats of the promoter fragment. Four tandem repeats of the promoter fragments were generated similarly. The -46 Cauliflower mosaic virus $35 \mathrm{~S}$ minimal promoter [45] was amplified by PCR with XhoI and BamHI sites attached to the 5'- and 3'-ends, 
respectively, and fused with four tandem repeats of EAS4 promoter fragment using $\mathrm{XhoI}$ site.

Promoter fragments with HindIII and BamHI sites attached to the $5^{\prime}$ - and $3{ }^{\prime}$-ends, respectively, were cloned into the corresponding sites of a pBE2113-GUS vector [46] to replace the $35 \mathrm{~S}$ promoter, producing a fusion of the promoter fragments with GUS. The construction of pBE2113-LUC and pBE2113-MEK2 ${ }^{\mathrm{DD}}$ has been described previously $[46,47]$.

\section{Prediction of cis-elements}

cis-Elements present in the EAS4 promoter were predicted using PLACE (https://www.dna.affrc.go.jp/PLACE/?action=newplace) [48], PlantCARE (http://bioinformatics. psb.ugent.be/webtools/plantcare/html/) [49] and PlantProm (http://linux1.softberry.com/berry.phtml?topic= plantprom\&group=data\&subgroup=plantprom) [50].

\section{Analysis of promoter activity}

Transformation, culture, and preparation of Agrobacterium (strain GV3101) cells were performed as described previously [51]. Agrobacterium cells $\left(\mathrm{OD}_{600}=0.1\right)$ carrying GUS driven by the respective promoters as a reporter were mixed with those carrying pBE2113-LUC as a control of Agrobacterium infection (GUS: LUC =9: 1), and then infiltrated into the leaves of $N$. benthamiana. After incubation at $25^{\circ} \mathrm{C}$ for $40-48 \mathrm{~h}$, leaf discs were excised from leaves using a cork borer (diameter $10 \mathrm{~mm}$ ). The leaf discs were floated on water and further incubated for $12 \mathrm{~h}$. Total RNA was extracted from leaf discs and converted to cDNA after DNase treatment using a PrimeScript RT reagent Kit with gDNA Eraser (Takara, Japan). To exclude transcripts accumulated in Agrobacterium cells, reversetranscription was performed with an oligo-dT primer. Transcript levels of GUS, LUC, and Nbactin 2 were quantified by qPCR, and the level of GUS transcript was doubly normalized to those of Nbactin2 and LUC.

For the expression of $M E K 2^{D D}$, Agrobacterium cells $\left(\mathrm{OD}_{600}=0.1\right)$ expressing GUS were mixed with those carrying pBE2113-LUC and those containing pBE2113$\operatorname{MEK}^{\mathrm{DD}}$ (GUS: LUC: MEK2 $\left.{ }^{\mathrm{DD}}=8: 1: 1\right)$, and then infiltrated into the leaves of $N$. benthamiana. After incubation at $25^{\circ} \mathrm{C}$ for $48 \mathrm{~h}$, total RNA was extracted, and used for RT-qPCR analysis.

\section{Production and purification of an anti-EAS antibody}

The peptide (QDENGKFKES) corresponding to residues 130-139 of EAS4 was synthesized and conjugated to keyhole limpet hemacyanin carrier by introducing a Cys residue to the $\mathrm{N}$-terminus of the peptide. Polyclonal antisera were raised in rabbits. Purification of antibodies was performed as follows. The coding sequence of EAS4 was amplified by PCR with NcoI and XhoI sites attached to the $5^{\prime}$ - and 3 '-ends, respectively, and cloned into the corresponding sites of a pET28a vector (Merck, Germany), allowing the production of C-terminal $\mathrm{His}_{6}-$ tagged EAS4 (EAS4-His). The resulting construct was used to transform E. coli strain Rosetta2(DE3) (Merck, Germany). Expression of the recombinant protein was induced by adding $0.1 \mathrm{mM}$ IPTG at $20^{\circ} \mathrm{C}$ overnight and purified with a 1-ml HisTrap HP column (GE Healthcare, USA) in accordance with the manufacturer's recommendations. Purified EAS4-His protein $(\sim 3 \mathrm{mg})$ was coupled to a 1-ml HiTrap NHS-activated HP column (GE Healthcare, USA) in accordance with the manufacturer's recommendations. Anti-EAS antiserum was applied to the column and washed extensively with buffer (20 mM Tris- $\mathrm{HCl}, \mathrm{pH} 7.5,1 \mathrm{M} \mathrm{NaCl}$ and $1 \%$ Triton X100). Bound antibodies were eluted with $0.1 \mathrm{M}$ Glycine$\mathrm{HCl}, \mathrm{pH} 2.5$, immediately neutralized and concentrated using an Amicon Ultra-4 (Merck, Germany).

\section{Protein extraction and immunoblotting analysis}

Protein extracts from tobacco leaves were prepared by grinding them in 5 volumes of buffer $[50 \mathrm{mM}$ Tris- $\mathrm{HCl}$, $\mathrm{pH} 7.5,150 \mathrm{mM} \mathrm{NaCl}, 5 \mathrm{mM}$ EDTA, $5 \mathrm{mM}$ DTT, and Complete protease inhibitor cocktail (Roche Applied Science)]. Supernatants were cleared by centrifugation at $21,500 \times g$ for $15 \mathrm{~min}$ at $4{ }^{\circ} \mathrm{C}$, and concentration of the protein extracts was determined using a Bio-Rad protein assay kit (Bio-Rad Laboratories, USA) with bovine gamma-globulin as the standard.

For immunoblotting analyses, proteins were separated by SDS-PAGE and transferred to polyvinylidene difluoride membranes (Merck, Germany). After blocking with 5\% nonfat dry milk, membranes were probed with $0.1 \mu \mathrm{g} / \mathrm{ml}$ anti-EAS antibody diluted with Western BLoT Immuno Booster (Takara, Japan) at $4{ }^{\circ} \mathrm{C}$ overnight. After washing, the membranes were incubated with horseradish peroxidase-labeled secondary antibody diluted with $1 \%$ nonfat dry milk at room temperature for $1 \mathrm{~h}$. The antigenantibody complexes were visualized using Western BLoT Hyper HRP Substrate (Takara, Japan).

\section{Capsidiol measurement}

The extraction and quantification of capsidiol were performed as described previously [52].

\section{Supplementary information}

Supplementary information accompanies this paper at https://doi.org/10. 1186/s12870-019-2204-1.

Additional file 1: Figure S1. Mevalonate and MEP pathways. Shown is a schematic representation of the mevalonate and MEP pathways. In the mevalonate pathway, IPP is synthesized from acetyl-CoA, whereas it is produced from pyruvate and G3P in the MEP pathway present in plastids. Abbreviations for chemicals and enzymes in the mevalonate pathway are as follows: Acetyl-CoA, acetyl coenzyme A; AACT, acetoacetyl-CoA thiolase; HMG-CoA, 3-hydroxy-3-methylglutaryl-CoA; HMGR, HMG-CoA 
reductase; HMGS, HMG-CoA synthase; MVK, mevalonate kinase: MVP. mevalonate-5-phosphate; MVPK, MVP kinase; MVPP, mevalonate-5diphosphate; MPD, MVPP decarboxylase; IPP, isopentenyl diphosphate; DMAPP, dimethylallyl diphosphate; IDI, IPP isomerase; FPP, farnesyl diphosphate; FPS, FPP synthase; SQS, squalene synthase; EAH, 5-epiaristolochene 1,3-dihydroxylase; EAS, 5-epi-aristolochene synthase. Abbreviations for chemicals and enzymes in the MEP pathway are as follows: G3P, glyceraldehyde 3-phosphate; DXP, 1-deoxy-D-xylulose 5-phosphate; DXR, DXP reductoisomerase; DXS, DXP synthase; MEP, 2-C-methyl-Derythritol 4-phosphate; CMS, MEP cytidylyltransferase; CDP-ME, 4-(cytidine 5'-diphospho)-2-C-methyl-D-erythritol; CDP-MEP, CDP-ME-2-phosphate; CMK, CDP-ME kinase; MECPP, 2-C-methyl-D-erythritol 2,4-cyclodiphosphate; MCS, MECPP synthase; HMBPP, 4-hydroxy-3-methylbut-2-enyl diphosphate; HDR, HMBPP reductase; HDS, HMBPP synthase; GGPP, geranylgeranyl diphosphate; GGPS, GGPP synthase; GPP, geranyl diphosphate; GPS, GPP synthase.

Additional file 2: Table S1. List of transcripts down-regulated in WS3 Additional file 3: Table S2. BLASTX analysis of transcripts downregulated in WS3

Additional file 4: Figure S2. Transcript levels of the capsidiol synthesis genes in another line of WIPK/SIPK-suppressed plants. Leaves of the vector control (V1) and WIPK/SIPK-suppressed plants (WS3 and WS5) were wounded, and harvested at the times indicated after wounding. Transcript levels of the genes were quantified by RT-qPCR and normalized to the level of Actin2 as an internal standard. Values are means with standard deviations of three to six biological replicates.

Additional file 5: Figure S3. Transcript levels of MEP genes over a time course after wounding. Leaves of the vector control (V1) and WIPK/SIPKsuppressed plants (WS3) were wounded, and harvested at the times indicated after wounding. Transcript levels of MEP genes were quantified by RT-qPCR and normalized to the level of Actin2 as an internal standard. Values are means with standard deviations of three biological replicates.

Additional file 6: Figure S4. Transcript levels of GUS fused to the 5'untranslated region of EAS4 or a 355 minimal promoter. Agrobacterium cells carrying GUS fused to the EAS4 promoter fragments, 5'-untranslated region of EAS4 or a 355 minimal promoter were mixed with those carrying LUC driven by a $35 S$ promoter, and infiltrated into $N$. benthamiana leaves. At 40-48 h after infiltration, the leaves were wounded, and harvested at the times indicated after wounding. Transcript levels of GUS, LUC, and Nbactin2 were quantified by RT-qPCR, and the level of GUS was doubly normalized to the levels of Nbactin2 and LUC as internal and infection standards, respectively. Values are means with standard deviations of three biological replicates.

Additional file 7: Figure S5. Expression of EAS is induced by three different methods of wounding. Leaves of the wild-type tobacco were wounded by three different methods. Hole, one or four small holes per a $1-\mathrm{cm}$ diameter circle were made in the leaves by pricking with a 10- $\mathrm{kl}$ tip. Crush, leaves were held with forceps strongly. Disc, discs were excised from the leaves and floated on water. The samples were harvested at $9 \mathrm{~h}$ after wounding, and the transcript levels of EAS were quantified by RTqPCR, and their levels were normalized to the levels of Actin2.

Additional file 8: Figure S6. Nucleotide sequences of regions -151 to - 85 of the EAS4 promoter. Nucleotide sequences of regions - 149 to 140 and -96 to -87 of the EAS4 promoter are similar to each other in opposite directions. The identical sequences are shown in red and blue, respectively.

Additional file 9: Table S3. List of primers used for QPCR analysis, and deletion and substitutions of the EAS4 promoter

\section{Abbreviations}

EAH: 5-epi-aristolochene 1,3-dihydroxylase; EAS: 5-epi-aristolochene synthase; ERF: Ethylene response factor; FPP: Farnesyl diphosphate; FPS: FPP synthase; GUS: $\beta$-glucuronidase; HMGR: 3-hydroxy-3-methylglutaryl-CoA reductase; IDI: IPP isomerase; IPP: Isopentenyl diphosphate; JA: Jasmonic acid; LUC: Luciferase; MAMP: Microbe-associated molecular pattern; MAPK: Mitogen-activated protein kinase; MEP: 2-C-methyl-D-erythritol 4phosphate; RT-qPCR: Reverse transcription-quantitative PCR; SQS: Squalene synthase; UTR: Untranslated region

\section{Acknowledgements}

The authors thank Sophien Kamoun for providing the pFB53 vector, Ichiro Mitsuhara for the pBE2113-GUS, PBE2113-LUC and PBE2113-MEK2 ${ }^{\mathrm{DD}}$ vectors, and Shigemi Seo for the SIPK-, WIPK-, and WIPK-and-SIPK-silenced tobacco plants. We also thank Hirofumi Yoshioka, Takeshi Hosaka, Takeshi Shimosato, and Katsuharu Saito for their technical and statistical assistance.

\section{Authors' contributions}

SK conceived and designed the research. SK, TK, NA, SH, TH, and YM performed the experiments. SK, TK, NA, SH, TH, and DT analyzed the data. SK and DT wrote the manuscript. All authors read and approved the final manuscript.

\section{Funding}

This work was supported by the Japan Society for the Promotion of Science KAKENHI [grant Nos. 21880020, 23688005, 17 K07665 to S.K.]; the Ministry of Education and Science, Japan [the Program for Dissemination of the TenureTrack System (to S.K.)]. The funding bodies were not involved in designing of research, collection, analysis, interpretation of data, or writing of the manuscript.

\section{Availability of data and materials}

Microarray data that support the findings of this study have been deposited in GEO repository with the accession code GSE133681. The other datasets used and/or analyzed during the current study are available from the corresponding author on reasonable request.

\section{Ethics approval and consent to participate}

Not applicable.

\section{Consent for publication}

Not applicable.

\section{Competing interests}

The authors declare that they have no competing interests.

\section{Author details}

${ }^{1}$ Faculty of Agriculture, Shinshu University, Nagano 399-4598, Japan.

${ }^{2}$ Graduate School of Bioagricultural Sciences, Nagoya University, Nagoya, Aichi 464-8601, Japan.

Received: 1 August 2019 Accepted: 15 December 2019 Published online: 21 December 2019

\section{References}

1. Freeman BC, Beattie GA. An overview of plant defenses against pathogens and herbivores. Plant Health Instructor. 2008. https://doi.org/10.1094/PHI-I2008-0226-01.

2. Jones JDG, Dangl JL. The plant immune system. Nature. 2006;444:323-9.

3. Ahuja I, Kissen R, Bones AM. Phytoalexins in defense against pathogens. Trends Plant Sci. 2012;17:73-90.

4. Rodríguez-Concepción M, Boronat A. Breaking new ground in the regulation of the early steps of plant isoprenoid biosynthesis. Curr Opin Plant Biol. 2015;25:17-22.

5. Widmann C, Gibson S, Jarpe MB, Johnson GL. Mitogen-activated protein kinase: conservation of a three-kinase module from yeast to human. Physiol Rev. 1999;79:143-80.

6. MAPK Group. Mitogen-activated protein kinase cascades in plants: a new nomenclature. Trends Plant Sci. 2002;7:301-8.

7. Ren D, Liu Y, Yang KY, Han L, Mao G, Glazebrook J, et al. A fungalresponsive MAPK cascade regulates phytoalexin biosynthesis in Arabidopsis. Proc Natl Acad Sci U S A. 2008;105:5638-43.

8. Mao G, Meng X, Liu Y, Zheng Z, Chen Z, Zhang S. Phosphorylation of a WRKY transcription factor by two pathogen-responsive MAPKs drives phytoalexin biosynthesis in Arabidopsis. Plant Cell. 2011;23:1639-53.

9. Kishi-Kaboshi M, Okada K, Kurimoto L, Murakami S, Umezawa T, Shibuya N, et al. A rice fungal MAMP-responsive MAPK cascade regulates metabolic flow to antimicrobial metabolite synthesis. Plant J. 2010;63:599-612.

10. Yang KY, Liu Y, Zhang S. Activation of a mitogen-activated protein kinase pathway is involved in disease resistance in tobacco. Proc Natl Acad Sci U S A. 2001;98:741-6. 
11. Ishihama N, Yamada R, Yoshioka M, Katou S, Yoshioka H. Phosphorylation of the Nicotiana benthamiana WRKY8 transcription factor by MAPK functions in the defense response. Plant Cell. 2011;23:1153-70.

12. Seo S, Katou S, Seto H, Gomi K, Ohashi Y. The mitogen-activated protein kinases WIPK and SIPK regulate the levels of jasmonic and salicylic acids in wounded tobacco plants. Plant J. 2007:49:899-909.

13. Wu J, Hettenhausen C, Meldau S, Baldwin IT. Herbivory rapidly activates MAPK signaling in attacked and unattacked leaf regions but not between leaves of Nicotiana attenuata. Plant Cell. 2007;19:1096-122.

14. Niki T, Mitsuhara I, Seo S, Ohtsubo N, Ohashi Y. Antagonistic effect of salicylic acid and jasmonic acid on the expression of pathogenesis-related (PR) protein genes in wounded mature tobacco leaves. Plant Cell Physiol. 1998;39:500-7.

15. Sato $C$, Seto $Y$, Nabeta $K$, Matsuura $H$. Kinetics of the accumulation of jasmonic acid and its derivatives in systemic leaves of tobacco (Nicotiana tabacum cv. Xanthi nc) and translocation of deuterium-labeled jasmonic acid from the wounding site to the systemic site. Biosci Biotechnol Biochem. 2009;73:1962-70.

16. Bevan M, Bancroft I, Bent E, Love K, Goodman H, Dean C, et al. Analysis of 1. $9 \mathrm{Mb}$ of contiguous sequence from chromosome 4 of Arabidopsis thaliana. Nature. 1998;391:485-8.

17. Hemmerlin A, Hoeffler JF, Meyer O, Tritsch D, Kagan IA, GrosdemangeBilliard C, et al. Cross-talk between the cytosolic mevalonate and the plastidial methylerythritol phosphate pathways in tobacco bright Yellow-2 cells. J Biol Chem. 2003;278:26666-76.

18. Opitz S, Nes WD, Gershenzon J. Both methylerythritol phosphate and mevalonate pathways contribute to biosynthesis of each of the major isoprenoid classes in young cotton seedlings. Phytochemistry. 2014;98:110-9.

19. Yin S, Mei L, Newman J, Back K, Chappell J. Regulation of sesquiterpene cyclase gene expression. Plant Physiol. 1997;115:437-51.

20. Newman JD, Yin S, Chappell J. Characterization of the TAC box, a ciselement within an elicitor-inducible sesquiterpene cyclase promoter. Plant J. 1998;16:1-12.

21. Kobayashi M, Ishihama N, Yoshioka H, Takabatake R, Tsuda S, Seo S, et al. Analyses of the cis-regulatory regions responsible for the transcriptional activation of the $N$ resistance gene by Tobacco mosaic virus. J Phytopathol. 2010;158:826-8

22. Li G, Meng X, Wang R, Mao G, Han L, Liu Y, et al. Dual-level regulation of ACC synthase activity by MPK3/MPK6 cascade and its downstream WRKY transcription factor during ethylene induction in Arabidopsis. PLoS Genet. 2012;8:e1002767.

23. Adachi H, Nakano T, Miyagawa N, Ishihama N, Yoshioka M, Katou Y, et al. WRKY transcription factors phosphorylated by MAPK regulate a plant immune NADPH oxidase in Nicotiana benthamiana. Plant Cell. 2015;27:2645-63.

24. Kamoun $S$, van West $P$, de Jong AJ, de Groot KE, Vleeshouwers VGAA, Govers F. A gene encoding a protein elicitor of Phytophthora infestans is down-regulated during infection of potato. Mol Plant-Microbe Interact. 1997;10:13-20.

25. Darvill AG, Albersheim P. Phytoalexins and their elicitors-a defense against microbial infection in plants. Annu Rev Plant Physiol. 1984;35:243-75.

26. Shibata Y, Ojika M, Sugiyama A, Yazaki K, Jones DA, Kawakita K, et al. The full-size ABCG transporters Nb-ABCG1 and Nb-ABCG2 function in pre- and postinvasion defense against Phytophthora infestans in Nicotiana benthamiana. Plant Cell. 2016;28:1163-81.

27. Han L, Li GJ, Yang KY, Mao G, Wang R, Liu Y, et al. Mitogen-activated protein kinase 3 and 6 regulate Botrytis cinerea-induced ethylene production in Arabidopsis. Plant J. 2010;64:114-27.

28. Kurusu T, Hamada J, Nokajima H, Kitagawa Y, Kiyoduka M, Takahashi A, et al Regulation of microbe-associated molecular pattern-induced hypersensitive cell death, phytoalexin production, and defense gene expression by calcineurin B-like protein-interacting protein kinases, OsCIPK14/15, in rice cultured cells. Plant Physiol. 2010;153:678-92.

29. Shibata Y, Kawakita K, Takemoto D. Age-related resistance of Nicotiana benthamiana against hemibiotrophic pathogen Phytophthora infestans requires both ethylene- and salicylic acid-mediated signaling pathways. Mol Plant-Microbe Interact. 2010;23:1130-42.

30. Takemoto D, Shibata Y, Ojika M, Mizuno Y, Imano S, Ohtsu M, et al. Resistance to Phytophthora infestans: exploring genes required for disease resistance in Solanaceae plants. J Gen Plant Pathol. 2018;84:312-20.

31. Singh AK, Kumar SR, Dwivedi V, Rai A, Pal S, Shasany AK, et al. A WRKY transcription factor from Withania somnifera regulates triterpenoid withanolide accumulation and biotic stress tolerance through modulation of phytosterol and defense pathways. New Phytol. 2017;215:1115-31.

32. Zang D, Wang J, Zhang X, Liu Z, Wang Y. Arabidopsis heat shock transcription factor HSFA7b positively mediates salt stress tolerance by binding to an E-box-like motif to regulate gene expression. J Exp Bot. 2019;70:5355-74.

33. Song N, Ma L, Wang W, Sun H, Wang L, Baldwin IT, et al. An ERF2-like transcription factor regulates production of the defense sesquiterpene capsidiol upon Alternaria alternate infection. J Exp Bot. 2019;70:5895-908.

34. Chassot C, Buchala A, Schoonbeek HJ, Métraux JP, Lamotte O. Wounding of Arabidopsis leaves causes a powerful but transient protection against Botrytis infection. Plant J. 2008;55:555-67.

35. Felix G, Duran JD, Volko S, Boller T. Plants have a sensitive perception system for the most conserved domain of bacterial flagellin. Plant J. 1999;18:265-76.

36. Choi D, Ward BL, Bostock RM. Differential induction and suppression of potato 3-hydroxy-3-methylglutaryl coenzyme a reductase genes in response to Phytophthora infestans and to its elicitor arachidonic acid. Plant Cell. 1992:4:1333-44

37. Yoshioka H, Yamada N, Doke N. cDNA cloning of sesquiterpene cyclase and squalene synthase, and expression of the genes in potato tuber infected with Phytophthora infestans. Plant Cell Physiol. 1999:40:993-8.

38. Yang $Q$, Trinh HX, Imai S, Ishihara A, Zhang L, Nakayashiki $H$, et al. Analysis of the involvement of hydroxyanthranilate hydroxycinnamoyltransferase and caffeoyl-CoA 3-O-methyltransferase in phytoalexin biosynthesis in oat. Mol Plant-Microbe Interact. 2004;17:81-9.

39. Keller H, Czernic P, Ponchet M, Ducrot PH, Back K, Chappell J, et al. Sesquiterpene cyclase is not a determining factor for elicitor- and pathogen-induced capsidiol accumulation in tobacco. Planta. 1998:205:467-76.

40. Wilkie GS, Dickson KS, Gray NK. Regulation of mRNA translation by $5^{\prime}$ - and 3'-UTR-binding factors. Trends Biochem Sci. 2003;28:182-8.

41. Xu G, Greene GH, Yoo H, Liu L, Marqués J, Motley J, et al. Global translational reprogramming is a fundamental layer of immune regulation in plants. Nature. 2017;545:487-90.

42. Merchante C, Brumos J, Yun J, Hu Q, Spencer KR, Enríquez P, et al. Genespecific translation regulation mediated by the hormone-signaling molecule EIN2. Cell. 2015:163:684-97.

43. Ohtsu M, Shibata Y, Ojika M, Tamura K, Hara-Nishimura I, Mori H, et al. Nucleoporin 75 is involved in the ethylene-mediated production of phytoalexin for the resistance of Nicotiana benthamiana to Phytophthora infestans. Mol Plant-Microbe Interact. 2014:27:1318-30.

44. Katou S, Asakura N, Kojima T, Mitsuhara I, Seo S. Transcriptome analysis of WIPK/SIPK-suppressed plants reveals induction by wounding of disease resistance-related genes prior to the accumulation of salicylic acid. Plant Cell Physiol. 2013;54:1005-15.

45. Odell JT, Nagy F, Chua NH. Identification of DNA sequences required for activity of the cauliflower mosaic virus 355 promoter. Nature. 1985; 313:810-2.

46. Mitsuhara I, Ugaki M, Hirochika H, Ohshima M, Murakami T, Gotoh Y, et al. Efficient promoter cassettes for enhanced expression of foreign genes in dicotyledonous and monocotyledonous plants. Plant Cell Physiol. 1996:37:49-59.

47. Takabatake R, Ando Y, Seo S, Katou S, Tsuda S, Ohashi Y, et al. MAP kinases function downstream of HSP90 and upstream of mitochondria in TMV resistance gene $\mathrm{N}$-mediated hypersensitive cell death. Plant Cell Physiol. 2007:48:498-510.

48. Higo K, Ugawa Y, Iwamoto M, Korenaga T. Plant cis-acting regulatory DNA elements (PLACE) database: 1999. Nucleic Acids Res. 1999:27:297-300.

49. Lescot $M$, Déhais $P$, Thijs $G$, Marchal $K$, Moreau $Y$, Van de Peer $Y$, et al. PlantCARE, a database of plant cis-acting regulatory elements and a portal to tools for in silico analysis of promoter sequences. Nucleic Acids Res. 2002; 30:325-7.

50. Shahmuradov IA, Gammerman AJ, Hancock JM, Bramley PM, Solovyev W. PlantProm: a database of plant promoter sequences. Nucleic Acids Res. 2003;31:114-7

51. Yokoo S, Inoue S, Suzuki N, Amakawa N, Matsui $H$, Nakagami H, et al. Comparative analysis of plant isochorismate synthases reveals structural mechanisms underlying their distinct biochemical properties. Biosci Rep. 2018:38:BSR20171457. 
52. Matsukawa M, Shibata Y, Ohtsu M, Mizutani A, Mori H, Wang P, et al. Nicotiana benthamiana calreticulin $3 a$ is required for the ethylenemediated production of phytoalexins and disease resistance against oomycete pathogen Phytophthora infestans. Mol Plant-Microbe Interact. 2013;26:880-92.

\section{Publisher's Note}

Springer Nature remains neutral with regard to jurisdictional claims in published maps and institutional affiliations.

Ready to submit your research? Choose BMC and benefit from:

- fast, convenient online submission

- thorough peer review by experienced researchers in your field

- rapid publication on acceptance

- support for research data, including large and complex data types

- gold Open Access which fosters wider collaboration and increased citations

- maximum visibility for your research: over $100 \mathrm{M}$ website views per year

At BMC, research is always in progress.

Learn more biomedcentral.com/submissions 